\title{
Averaging Operators Along a Certain Type of Surfaces with Hypersingularity
}

\author{
Jin Bong Lee*, Jongho Lee and Chan Woo Yang
}

\begin{abstract}
In this paper we obtain almost sharp decay estimates for $L^{2}$ operator norm of strongly singular oscillatory integral operators in $\mathbb{R}^{n+1}$ for $n \geq 2$; we prove some necessary condition for $L^{2}$ estimates. Also, we prove that the operators are bounded on $L^{p}$ for some $p \neq 2$ and the range of $p$ depends on the hypersingularity of the operators.
\end{abstract}

\section{Introduction}

The origin of the hypersingular integral operators along curves is the Hilbert transform along curves:

$$
\mathcal{H}_{\mathcal{C}} f(x)=\mathrm{p} \cdot \mathrm{v} \cdot \int_{\mathbb{R}} f(x-\mathcal{C}(s)) \frac{d s}{s}, \quad x \in \mathbb{R}^{n}
$$

with an appropriate curve $\mathcal{C}(s)=(s, c(s))$ in $\mathbb{R}^{n}$. It is a very well-known fact due to E. M. Stein and S. Wainger [5] that the Hilbert transform along curves is bounded operator on $L^{p}$ with $1<p<\infty$, when one takes the well-curved $\mathcal{C}$ as an appropriate curve. That is, one chooses $\mathcal{C}(s)=(s, c(s))$ with a smooth $c$ in $\mathbb{R}^{n-1}$, and $c(0)=0$ so that $\left\langle\left\{\left.\frac{d^{k} \mathcal{C}(s)}{d s^{k}}\right|_{s=0}\right\}_{k=1,2,3, \ldots}\right\rangle=\mathbb{R}^{n}$.

One easily notices that above operator does not contain any oscillating terms. It is S. Chandarana [1] who first tried to control this operator with an additional oscillating term, $\frac{e^{-2 \pi i|s|^{-\beta}}}{|s|^{\alpha}}$, since with the singular term such as $\frac{1}{|s|^{\alpha}}$ the operator is not bounded on $L^{2}$. In his paper, the hypersingular integral is defined as

$$
\mathcal{T}_{\alpha, \beta} f(x, y)=\mathrm{p} \cdot \mathrm{v} \cdot \int_{[-1,1]} f(x-s, y-c(s)) e^{-2 \pi i|s|^{-\beta}} \frac{d s}{s|s|^{\alpha}}, \quad \alpha, \beta>0
$$

with $c(s)=|s|^{k}$ or $c(s)=|s|^{k} \operatorname{sgn}(s)$ and $k \geq 2$. Then $\mathcal{T}_{\alpha, \beta}$ is bounded on (i) $L^{2}$ if and only if $\beta \geq 3 \alpha$, and (ii) $L^{p}$ with $1+\frac{3 \alpha(\beta+1)}{\beta(\beta+1)+(\beta-3 \alpha)}<p<1+\frac{\beta(\beta+1)+(\beta-3 \alpha)}{3 \alpha(\beta+1)}$ if $\beta>3 \alpha$.

In the same direction, N. Laghi and N. Lyall 3] extended this result to well-curved $\mathcal{C}$ which was considered by E. M. Stein and S. Wainger. That is, with $\mathcal{T}_{\alpha, \beta}=\mathcal{T}_{\mathcal{C}}$, they proved Received April 3, 2019; Accepted October 31, 2019.

Communicated by Duy-Minh Nhieu.

2010 Mathematics Subject Classification. 42B20, 42B15.

Key words and phrases. singular integrals along surfaces, oscillatory integrals, hypersingularity, Bessel functions.

*Corresponding author. 
that if $\mathcal{C}(s)$ is well-curved, then (i) $\mathcal{T}_{\mathcal{C}}$ is bounded on $L^{2}\left(\mathbb{R}^{d}\right)$ if and only if $\alpha \leq \beta /(d+1)$, and (ii) with $\alpha=0$ and $\beta>0, \mathcal{T}_{\mathcal{C}}: L \log L\left(\mathbb{R}^{d}\right) \rightarrow L^{1, \infty}\left(\mathbb{R}^{d}\right)$.

Recently, X. Wu and X. Yu [6] studied the case when $\mathcal{C}(s)=\Gamma_{\theta}(s)$ is a homogeneous curve given by $\Gamma_{\theta}(s)=\left(\theta_{1}|s|^{p_{1}}, \ldots, \theta_{d}|s|^{p_{d}}\right)$ or $\Gamma_{\theta}(s)=\operatorname{sgn}(s)\left(\theta_{1}|s|^{p_{1}}, \ldots, \theta_{d}|s|^{p_{d}}\right)$ for $\theta=\left(\theta_{1}, \ldots, \theta_{d}\right) \in \mathbb{R}^{d}$. They proved that the corresponding operator, $T_{d, \alpha, \beta} f(x)=$ p.v. $\int_{-1}^{1} f\left(x-\Gamma_{\theta}(s)\right) \frac{e^{-2 \pi i|t|^{-\beta}}}{s|s|^{\alpha}} d s$, is bounded on the $\alpha$-modulation spaces such as the inhomogeneous Besov spaces.

Now, returning to the work of S. Chandarana [1], we consider a natural question of varying $\mathcal{C}(s)$ so that it is no longer a curve but a surface with some curvature conditions. So, it is natural to change the dimension $(n \geq 2)$ and a domain $\left([-1,1] \rightarrow \mathbb{R}^{n}\right)$. Also, we simply set $\Gamma(t)=\left(t,|t|^{k}\right)$, where $t=\left(t_{1}, \ldots, t_{n}\right)$ and $k \geq 1$ so that it represents the case of hypersurface, co-dimesion 1 . That is, we are interested in operators of the form

$$
\mathcal{R} f(x)=\int_{\mathbb{R}^{n}} f(x-\Gamma(t)) \frac{e^{-2 \pi i|t|^{-\beta}} \Omega(t)}{|t|^{\alpha+n}} d t, \quad \Gamma(t)=\left(t,|t|^{k}\right), \quad t=\left(t_{1}, \ldots, t_{n}\right),
$$

where the kernel $\Omega(t) /|t|^{n}$ satisfies the following conditions:

(1) it is homogeneous of degree $-n$;

(2) it is the class $C^{\infty}\left(\mathbb{R}^{n}-\{0\}\right)$;

(3) $\int_{|t|=1} \Omega(t) d \sigma(t)=0$.

Our main results are

Theorem 1.1. For $n \geq 2$,

(1) $\mathcal{R}$ is bounded on $L^{2}\left(\mathbb{R}^{n+1}\right)$ if $\beta>2 \alpha>0$. Conversely, $\mathcal{R}$ is not bounded on $L^{2}\left(\mathbb{R}^{n+1}\right)$ if $\beta<2 \alpha$. The case $\beta=2 \alpha$ is still open.

(2) $\mathcal{R}$ is bounded of $L^{p}\left(\mathbb{R}^{n+1}\right)$ for $\alpha / \beta<1 / p<(\beta-\alpha) / \beta$ if $\beta>2 \alpha$.

Note that the statement of Theorem 1.1 holds uniformly for the class of the kernels of the operator $\mathcal{R}$. That is, the necessary condition asserts that one can find a certain kernel of which the associated operator is not bounded on $L^{2}$ when $\beta<2 \alpha$.

Outline of proof: We will prove the $L^{2}$ estimate in the following steps. First, we decompose the Fourier multiplier of $\mathcal{R}$ so that the corresponding phase function has bounded below partial derivatives. Second, we obtain decay estimates on each decomposed component of the Fourier multiplier by making use of the Van der Corput lemma and the stationary phase method. Then, by interpolation with a simple size estimate, we get the $L^{p}$ estimate. For the converse statement of the main theorem, we use the Bessel function 
to simplify the multiplier of $\mathcal{R}$ and figure out that the multiplier blows up along a certain curve.

Throughout this paper, the symbol $\lesssim$ implies that the inequality holds with some harmless constant multiplied on the right-hand side, and $\left|S^{n-2}\right|$ denotes the measure of the $(n-2)$-dimensional sphere embedded in $n$-dimensional Euclidean space $\mathbb{R}^{n}$.

\section{Preliminaries}

In this section, we introduce some well-known properties of oscillatory integrals used in our proofs. We will use these properties as lemmas.

Lemma 2.1 (Van der Corput's lemma). [4, Corollary, p. 334] Suppose $\phi$ is real-valued, smooth in $(a, b)$, and $\left|\phi^{(k)}(x)\right| \geq C>0$ for all $x \in(a, b)$. Then

$$
\left|\int_{a}^{b} e^{i \lambda \phi(x)} \psi(x) d x\right| \leq c_{k} \lambda^{-1 / k}\left[|\psi(b)|+\int_{a}^{b}\left|\psi^{\prime}(x)\right| d x\right]
$$

for $\psi \in C_{0}^{\infty}((a, b))$ when (i) $k \geq 2$, or (ii) $k=1$ and $\phi^{\prime}(x)$ is monotonic. Note that $c_{k}$ is independent of $\phi, \psi$, and $\lambda$.

Lemma 2.2 (Asymptotic integrals). [4, Proposition 3, p. 334] Suppose $k \geq 2$ and $\phi\left(x_{0}\right)=$ $\cdots=\phi^{(k-1)}\left(x_{0}\right)=0 \neq \phi^{(k)}\left(x_{0}\right)$. Then

$$
\int e^{i \lambda \phi(x)} \psi(x) d x \sim \lambda^{-1 / k} \quad \text { as } \lambda \rightarrow \infty
$$

when $\psi$ is supported in a sufficiently small neighborhood of $x_{0}$.

We also use some properties of the Bessel function,

$$
J_{m}(r)=\frac{1}{2 \pi} \int_{0}^{2 \pi} e^{i r \sin (\theta)} e^{-i m \theta} d \theta=\frac{(r / 2)^{m}}{\Gamma(m+1 / 2) \pi^{1 / 2}} \int_{-1}^{1} e^{i r t}\left(1-t^{2}\right)^{m-1 / 2} d t .
$$

The last equality holds when $m>-1 / 2$.

Lemma 2.3 (Recursion relation and asymptotic behavior of the Bessel function).

(1) $\frac{d}{d r}\left[r^{-m} J_{m}(r)\right]=-r^{-m} J_{m+1}(r)$,

(2) $J_{m}(r)=O\left(r^{-1 / 2}\right)$ as $r \rightarrow \infty$,

(3) $J_{m}(r) \sim r^{m}$ as $r \rightarrow 0$ for $\operatorname{Re}(m)>-1 / 2$.

For (1), (2) see E. M. Stein [4, (14), (15), (16), p. 338]. Also, for (3) see L. Grafakos [2, B.6, p. 429]. 


\section{3. $L^{2}$ estimates}

The multiplier $m$ of the operator $\mathcal{R}$ can be written as

$$
m(\xi)=\int_{\mathbb{R}^{n}} e^{-2 \pi i\left(|t|^{-\beta}+\xi^{\prime} \cdot t+\xi_{n+1}|t|^{k}\right)} \frac{\Omega(t)}{|t|^{n+\alpha}} d t,
$$

where $\xi^{\prime}=\left(\xi_{1}, \ldots, \xi_{n}\right)$. By using the spherical coordinate, the multiplier can be rewritten as

$$
m(\xi)=\int_{S^{n-2}} \int_{0}^{\pi} \int_{0}^{\infty} e^{-2 \pi i\left(r^{-\beta}+\left|\xi^{\prime}\right| r \cos (\theta)+\xi_{n+1} r^{k}\right)} r^{-\alpha-1} \Omega(\theta, \sigma)(\sin (\theta))^{n-2} d r d \theta d \sigma,
$$

where $\theta$ denotes the angle between $t$ and $\xi^{\prime}$. Then, in the $r$ variable, we decompose the multiplier as

$$
m_{l}(\xi)=\int_{S^{n-2}} \int_{0}^{\pi} \int_{0}^{\infty} e^{-2 \pi i g(r, \theta)} \eta\left(2^{l} r\right) r^{-\alpha-1} \Omega(\theta, \sigma)(\sin (\theta))^{n-2} d r d \theta d \sigma,
$$

where $g(r, \theta)=r^{-\beta}+\left|\xi^{\prime}\right| r \cos (\theta)+\xi_{n+1} r^{k}$, and $\eta(r)$ is a smooth function compactly supported in $[1 / 2,2]$. Next, make the change of variables $r \mapsto 2^{-l} r$ to write

$$
m_{l}(\xi)=2^{\alpha l} \int_{S^{n-2}} \int_{0}^{\pi} \int_{0}^{\infty} e^{-2 \pi i g\left(2^{-l} r, \theta\right)} \eta(r) r^{-\alpha-1} \Omega(\theta, \sigma)(\sin (\theta))^{n-2} d r d \theta d \sigma .
$$

With a slight abuse of notation, set

$$
g\left(2^{-l} r, \theta\right)=g(r, \theta)=2^{\beta l} r^{-\beta}+2^{-k l} \xi_{n+1} r^{k}+2^{-l}\left|\xi^{\prime}\right| r \cos (\theta) .
$$

Simple computations give

$$
\begin{aligned}
g_{r}^{\prime}(r, \theta) & =-\beta 2^{\beta l} r^{-\beta-1}+k 2^{-k l} \xi_{n+1} r^{k-1}+2^{-l}\left|\xi^{\prime}\right| \cos (\theta), \\
g_{\theta}^{\prime}(r, \theta) & =-2^{-l}\left|\xi^{\prime}\right| r \sin (\theta), \\
g_{r r}^{\prime \prime}(r, \theta) & =\beta(\beta+1) 2^{\beta l} r^{-\beta-2}+k(k-1) 2^{-k l} \xi_{n+1} r^{k-2}, \\
g_{\theta \theta}^{\prime \prime}(r, \theta) & =-2^{-l}\left|\xi^{\prime}\right| r \cos (\theta), \\
\lambda & =\max \left\{2^{\beta l}, 2^{-k l}\left|\xi_{n+1}\right|, 2^{-l}\left|\xi^{\prime}\right|\right\} .
\end{aligned}
$$

Lemma 3.1. Let $\epsilon=\beta 2^{-k-\beta-3}$.

(1) For all $(r, \theta) \in[1 / 2,2] \times[0, \pi]$,

$$
\max \left\{\left|g_{r}^{\prime}(r, \theta)\right|,\left|g_{\theta}^{\prime}(r, \theta)\right|,\left|g_{r r}^{\prime \prime}(r, \theta)\right|,\left|g_{\theta \theta}^{\prime \prime}(r, \theta)\right|\right\} \geq \epsilon \lambda .
$$

(2) If $\left|g_{r}^{\prime}\left(r_{0}, \theta_{0}\right)\right| \geq \epsilon \lambda$ for some $\left(r_{0}, \theta_{0}\right) \in[1 / 2,2] \times[0, \pi]$, then $\left|g_{r}^{\prime}(r, \theta)\right| \geq \frac{\epsilon}{2} \lambda$ for all $(r, \theta)$ satisfying

$$
\left|r-r_{0}\right| \leq \frac{2^{-2(\beta+k+4)}}{(\beta+1)^{3}+k^{3}}=\epsilon_{1} \quad \text { and } \quad\left|\theta-\theta_{0}\right| \leq \beta 2^{-k-\beta-7}=\epsilon_{2} .
$$

The same assertion holds for each $g_{\theta}^{\prime}, g_{r r}^{\prime \prime}, g_{\theta \theta}^{\prime \prime}$. 
Proof. To prove the first assertion, suppose that there exists $(r, \theta) \in[1 / 2,2] \times[0, \pi]$ such that

$$
\max \left\{\left|g_{r}^{\prime}(r, \theta)\right|,\left|g_{\theta}^{\prime}(r, \theta)\right|,\left|g_{r r}^{\prime \prime}(r, \theta)\right|,\left|g_{\theta \theta}^{\prime \prime}(r, \theta)\right|\right\}<\epsilon \lambda .
$$

Combining $(3.5)$ with 3.2 and $(3.3)$ to obtain

$$
2^{-2} 2^{-2 l}\left|\xi^{\prime}\right|^{2} \leq 2^{-2 l}\left|\xi^{\prime}\right|^{2} r^{2}\left(\sin ^{2}(\theta)+\cos ^{2}(\theta)\right)=\left|g_{\theta}^{\prime}(r, \theta)\right|^{2}+\left|g_{\theta \theta}^{\prime \prime}(r, \theta)\right|^{2} \leq 2 \epsilon^{2} \lambda^{2}
$$

which yields

$$
2^{-l}\left|\xi^{\prime}\right| \leq 2 \sqrt{2} \epsilon \lambda<\lambda
$$

Combining (3.5) with $(3.1)$ and $(3.3)$ to obtain

$$
\left|-\beta 2^{\beta l} r^{-\beta}+k 2^{-k l} \xi_{n+1} r^{k}\right|=\left|r g_{r}^{\prime}(r, \theta)+g_{\theta \theta}^{\prime \prime}(r, \theta)\right| \leq 3 \epsilon \lambda .
$$

Make use of (3.5) again to obtain

$$
\left|\beta(\beta+1) 2^{\beta l} r^{-\beta}+k(k-1) 2^{-k l} \xi_{n+1} r^{k}\right|=\left|r^{2} g_{r r}^{\prime \prime}(r, \theta)\right| \leq 4 \epsilon \lambda .
$$

Using (3.7) and (3.8) we obtain

$$
\begin{gathered}
2^{-k l}\left|\xi_{n+1}\right| \leq \frac{(7+3 \beta) \epsilon r^{-k}}{k(k+\beta)} \lambda \leq \frac{(7+3 \beta) \epsilon 2^{k}}{k(k+\beta)} \lambda<\lambda, \\
2^{\beta l} \leq \frac{(1+3 k) \epsilon r^{\beta}}{\beta(k+\beta)} \lambda \leq \frac{(1+3 k) \epsilon 2^{\beta}}{\beta(k+\beta)} \lambda<\lambda .
\end{gathered}
$$

By combining (3.4), (3.6), (3.9), and 3.10 we immediately obtain a contradiction. This completes the proof of the first assertion of the lemma.

To prove the second assertion of the lemma, thanks to the mean value theorem one can write

$$
\begin{aligned}
\left|g_{r}^{\prime}(r, \theta)\right| \geq & \left|g_{r}^{\prime}\left(r_{0}, \theta_{0}\right)\right|-\left|g_{r}^{\prime}(r, \theta)-g_{r}^{\prime}\left(r_{0}, \theta_{0}\right)\right| \\
\geq & \epsilon \lambda-\left|\beta(\beta+1) 2^{\beta l} r_{1}^{-\beta-2}\right|\left|r-r_{0}\right|-\left|k(k-1) 2^{-k l} \xi_{n+1} r_{1}^{k-2}\right|\left|r-r_{0}\right| \\
& -\left|2^{-l} \xi_{n+1}\right|\left|\sin \left(\theta_{1}\right)\right|\left|\theta-\theta_{0}\right| \\
\geq & \epsilon \lambda-\lambda \beta(\beta+1) 2^{\beta+2}\left|r-r_{0}\right|-\lambda k(k-1) 2^{k-2}\left|r-r_{0}\right|-\lambda\left|\theta-\theta_{0}\right| \\
\geq & \epsilon \lambda-2^{-3} \epsilon \lambda-2^{-3} \epsilon \lambda-2^{-3} \epsilon \lambda \geq \frac{\epsilon}{2} \lambda .
\end{aligned}
$$

The cases $g_{\theta}^{\prime}, g_{r r}^{\prime \prime}$, and $g_{\theta \theta}^{\prime \prime}$ can be treated in a similar way.

Let $\epsilon_{1}$ and $\epsilon_{2}$ be as in Lemma 3.1. Define an index set $\mathcal{I}$ by

$$
\mathcal{I}=\left\{\left(j_{1}, j_{2}\right) \in \epsilon_{1} \mathbb{Z} \times \epsilon_{2} \mathbb{Z}: \frac{1}{4} \leq j_{1} \leq 4 \text { and } 0 \leq j_{2} \leq 2 \pi\right\} .
$$


Decompose the multiplier $m_{l}$ as

$$
m_{l}(\xi)=\sum_{\boldsymbol{j} \in \mathcal{I}} m_{l, \boldsymbol{j}}(\xi)
$$

where for $\boldsymbol{j}=\left(j_{1}, j_{2}\right)$,

$$
m_{l, \boldsymbol{j}}(\xi)=2^{\alpha l} \int_{S^{n-2}} \int_{0}^{\pi} \int_{0}^{\infty} e^{i g(r, \theta)} r^{-\alpha-1} \chi_{\boldsymbol{j}}(r, \theta) \eta(r) \Omega(\theta, \sigma)(\sin (\theta))^{n-2} d r d \theta d \sigma
$$

with

$$
\chi_{\boldsymbol{j}}(r, \theta)=\chi_{1}\left(\frac{4\left(r-j_{1}\right)}{3 \epsilon_{1}}\right) \chi_{1}\left(\frac{4\left(\theta-j_{2}\right)}{3 \epsilon_{2}}\right),
$$

where $\operatorname{supp} \chi_{1} \subset[-1,1], \chi_{1} \equiv 1$ on $[-1 / 2,1 / 2]$. Therefore, $\chi_{j}$ is a smooth function equal to 1 in $\left[j_{1}-\frac{\epsilon_{1}}{4}, j_{1}+\frac{\epsilon_{1}}{4}\right] \times\left[j_{2}-\frac{\epsilon_{2}}{4}, j_{2}+\frac{\epsilon_{2}}{4}\right]$ and compactly supported in $\left[j_{1}-\frac{3 \epsilon_{1}}{4}, j_{1}+\frac{3 \epsilon_{1}}{4}\right] \times$ $\left[j_{2}-\frac{3 \epsilon_{2}}{4}, j_{2}+\frac{3 \epsilon_{2}}{4}\right]$; hence, the collection $\left\{\chi_{j}\right\}_{j}$ forms a partition of unity in the support of $\eta$.

Let $R_{\boldsymbol{j}}$ be the support of $\chi_{\boldsymbol{j}}$, and divide the index set $\mathcal{I}$ into four subsets $\mathcal{I}_{r}, \mathcal{I}_{\theta}, \mathcal{I}_{r r}$, and $\mathcal{I}_{\theta \theta}$ defined by

$$
\begin{gathered}
\mathcal{I}_{r}=\left\{\boldsymbol{j}=\left(j_{1}, j_{2}\right) \in \mathcal{I}:\left|g_{r}^{\prime}(r, \theta)\right| \geq \frac{\epsilon}{2} \lambda \text { for all }(r, \theta) \in R_{\boldsymbol{j}}\right\}, \\
\mathcal{I}_{\theta}=\left\{\boldsymbol{j}=\left(j_{1}, j_{2}\right) \in \mathcal{I}:\left|g_{\theta}^{\prime}(r, \theta)\right| \geq \frac{\epsilon}{2} \lambda \text { for all }(r, \theta) \in R_{\boldsymbol{j}}\right\}, \\
\mathcal{I}_{r r}=\left\{\boldsymbol{j}=\left(j_{1}, j_{2}\right) \in \mathcal{I}:\left|g_{r r}^{\prime}(r, \theta)\right| \geq \frac{\epsilon}{2} \lambda \text { for all }(r, \theta) \in R_{\boldsymbol{j}}\right\}, \\
\mathcal{I}_{\theta \theta}=\left\{\boldsymbol{j}=\left(j_{1}, j_{2}\right) \in \mathcal{I}:\left|g_{\theta \theta}^{\prime}(r, \theta)\right| \geq \frac{\epsilon}{2} \lambda \text { for all }(r, \theta) \in R_{\boldsymbol{j}}\right\} .
\end{gathered}
$$

Thanks to Lemma 3.1 it is clear that

$$
\mathcal{I}=\mathcal{I}_{r} \cup \mathcal{I}_{\theta} \cup \mathcal{I}_{r r} \cup \mathcal{I}_{\theta \theta}
$$

and without loss of generality we may assume that they are mutually disjoint. One may therefore write

$$
m_{l}(\xi)=\sum_{\boldsymbol{j} \in \mathcal{I}_{r}} m_{l, \boldsymbol{j}}(\xi)+\sum_{\boldsymbol{j} \in \mathcal{I}_{\theta}} m_{l, \boldsymbol{j}}(\xi)+\sum_{\boldsymbol{j} \in \mathcal{I}_{r r}} m_{l, \boldsymbol{j}}(\xi)+\sum_{\boldsymbol{j} \in \mathcal{I}_{\theta \theta}} m_{l, \boldsymbol{j}}(\xi) .
$$

Now we separately consider four cases: (i) $\boldsymbol{j} \in \mathcal{I}_{r}$; (ii) $\boldsymbol{j} \in \mathcal{I}_{\theta}$; (iii) $\boldsymbol{j} \in \mathcal{I}_{r r}$; (iv) $\boldsymbol{j} \in \mathcal{I}_{\theta \theta}$.

(i) $j \in \mathcal{I}_{r}$ : In this case, we use integration by parts of 3.11 with respect to $r$ to obtain

$$
\begin{gathered}
m_{l, \boldsymbol{j}}(\xi)=2^{\alpha l} \int_{S^{n-2}} \int_{0}^{\pi} \int_{0}^{\infty} e^{-2 \pi i g(r, \theta)} \frac{\partial}{\partial r}\left[\frac{-1}{i g_{r}^{\prime}(r, \theta)} r^{-\alpha-1} \chi_{\boldsymbol{j}}(r, \theta) \eta(r)\right] \Omega(\theta, \sigma) \\
\times(\sin (\theta))^{n-2} d r d \theta d \sigma,
\end{gathered}
$$


which gives

$$
\left|m_{l, j}(\xi)\right| \leq 2^{\alpha l}\left|S^{n-2}\right|\|\Omega\|_{L^{\infty}} 2^{\alpha+1} \lambda^{-1}=C(n, \alpha) 2^{\alpha l} \lambda^{-1},
$$

hence

$$
\left|m_{l, \boldsymbol{j}}(\xi)\right| \lesssim 2^{\alpha l} \lambda^{-1}
$$

There is also a trivial bound:

$$
\left|m_{l, j}(\xi)\right| \lesssim 2^{\alpha l}
$$

In view of the definition of $\lambda$ in (3.4), one has

$$
\left|m_{l, \boldsymbol{j}}(\xi)\right| \lesssim \begin{cases}2^{(\alpha-\beta) l} \leq 2^{(\alpha-\beta / 2) l} & \text { if } l \geq 0 \\ 2^{\alpha l} & \text { if } l<0\end{cases}
$$

(ii) $j \in \mathcal{I}_{\theta}$ : In this case we use integration by parts of 3.11 with respect to $\theta$ to obtain

$$
\begin{aligned}
m_{l, \boldsymbol{j}}(\xi)=2^{\alpha l} \int_{S^{n-2}} \int_{0}^{\pi} \int_{0}^{\infty} & e^{-2 \pi i g(r, \theta)} r^{-\alpha-1} \eta(r) \\
& \times \frac{\partial}{\partial \theta}\left[\frac{-1}{i g_{\theta}^{\prime}(r, \theta)} \chi_{\boldsymbol{j}}(r, \theta) \Omega(\theta, \sigma)(\sin (\theta))^{n-2}\right] d r d \theta d \sigma,
\end{aligned}
$$

which gives

$$
\left|m_{l, j}(\xi)\right| \leq 2^{\alpha l}\left|\sigma_{n-2}\right|\|\Omega\|_{L^{\infty}} 2^{\alpha+1} \lambda^{-1}=C(n, \alpha) 2^{\alpha l} \lambda^{-1},
$$

hence again

$$
\left|m_{l, j}(\xi)\right| \lesssim 2^{\alpha l} \lambda^{-1}
$$

Make use of the similar argument as above to obtain that if $j \in \mathcal{I}_{\theta}$, then

$$
\left|m_{l, \boldsymbol{j}}(\xi)\right| \lesssim \begin{cases}2^{(\alpha-\beta) l} \leq 2^{(\alpha-\beta / 2) l} & \text { if } l \geq 0 \\ 2^{\alpha l} & \text { if } l<0\end{cases}
$$

(iii) $\boldsymbol{j} \in \mathcal{I}_{r r}$ : In this case we apply the van der Corput lemma of the second order in the $r$ variable to obtain

$$
\left|m_{l, j}(\xi)\right| \lesssim 2^{\alpha l} \lambda^{-1 / 2}
$$

Make use of the similar argument as above to obtain that if $\boldsymbol{j} \in \mathcal{I}_{r r}$, then

$$
\left|m_{l, \boldsymbol{j}}(\xi)\right| \lesssim \begin{cases}2^{(\alpha-\beta / 2) l} & \text { if } l \geq 0 \\ 2^{\alpha l} & \text { if } l<0\end{cases}
$$

(iv) $\boldsymbol{j} \in \mathcal{I}_{\theta \theta}$ : In this case we apply the van der Corput lemma of the second order in the $\theta$ variable to obtain

$$
\left|m_{l, j}(\xi)\right| \lesssim 2^{\alpha l} \lambda^{-1 / 2}
$$


Make use of the similar argument as above to obtain that if $\boldsymbol{j} \in \mathcal{I}_{\theta \theta}$, then

$$
\left|m_{l, \boldsymbol{j}}(\xi)\right| \lesssim \begin{cases}2^{(\alpha-\beta) l} & \text { if } l \geq 0, \\ 2^{\alpha l} & \text { if } l<0 .\end{cases}
$$

By combining (3.12)-3.15) one finally obtains

$$
\begin{aligned}
& \left|m_{l}(\xi)\right| \\
\leq & \sum_{\boldsymbol{j} \in \mathcal{I}_{r}}\left|m_{l, \boldsymbol{j}}(\xi)\right|+\sum_{\boldsymbol{j} \in \mathcal{I}_{\theta}}\left|m_{l, \boldsymbol{j}}(\xi)\right|+\sum_{\boldsymbol{j} \in \mathcal{I}_{r r}}\left|m_{l, \boldsymbol{j}}(\xi)\right|+\sum_{\boldsymbol{j} \in \mathcal{I}_{\theta \theta}}\left|m_{l, \boldsymbol{j}}(\xi)\right| \lesssim \begin{cases}2^{(\alpha-\beta / 2) l} & \text { if } l \geq 0, \\
2^{\alpha l} & \text { if } l<0\end{cases}
\end{aligned}
$$

which yields

$$
\left\|\mathcal{R}_{l}\right\|_{L^{2} \rightarrow L^{2}} \lesssim \begin{cases}2^{(\alpha-\beta / 2) l} & \text { if } l \geq 0 \\ 2^{\alpha l} & \text { if } l<0\end{cases}
$$

One also has a size estimate

$$
\left\|\mathcal{R}_{l}\right\|_{L^{1} \rightarrow L^{1}} \lesssim 2^{\alpha l}
$$

By interpolating (3.16) and (3.17) we obtain

$$
\left\|\mathcal{R}_{l}\right\|_{L^{p} \rightarrow L^{p}} \lesssim \begin{cases}2^{(\alpha-\beta+\beta / p) l} & \text { if } l \geq 0 \\ 2^{\alpha l} & \text { if } l<0 .\end{cases}
$$

The right-hand side of $(3.18)$ is summable when $\alpha>0$ and $1 / p<(\beta-\alpha) / \beta$. By using the duality argument, for $\beta>2 \alpha>0$ and $\alpha / \beta<1 / p<(\beta-\alpha) / \beta$ we have

$$
\|\mathcal{R} f\|_{L^{p}\left(\mathbb{R}^{n+1}\right)} \lesssim \alpha, \beta\|f\|_{L^{p}\left(\mathbb{R}^{n+1}\right)} .
$$

Since we have an $L^{2}$ estimate for $\mathcal{R}$, by controlling the multiplier $m$, one can obtain the following Sobolev estimate. Consider two cases: (i) $\left|\xi_{3}\right| \geq\left|\xi^{\prime}\right|$ and (ii) $\left|\xi_{3}\right|<\left|\xi^{\prime}\right|$.

(i) $\left|\xi_{3}\right| \geq\left|\xi^{\prime}\right|:$

$$
\begin{aligned}
& \sum_{l \in \mathbb{Z}}\left|m_{l}(\xi)\right| \leq \sum_{l \in \mathbb{Z}} \sum_{\boldsymbol{j} \in \mathcal{I}_{r} \cup \mathcal{I}_{\theta}}\left|m_{l, j}(\xi)\right|+\sum_{l \in \mathbb{Z}} \sum_{\boldsymbol{j} \in \mathcal{I}_{r r} \cup \mathcal{I}_{\theta \theta}}\left|m_{l, j}(\xi)\right| \\
& \lesssim \sum_{j \in \mathcal{I}_{r} \cup \mathcal{I}_{\theta}} \sum_{l \in \mathbb{Z}} 2^{\alpha l} \lambda^{-1}+\sum_{\boldsymbol{j} \in \mathcal{I}_{r r} \cup \mathcal{I}_{\theta \theta}} \sum_{l \in \mathbb{Z}} 2^{\alpha l} \lambda^{-1 / 2} \\
& \lesssim \sum_{l \in \mathbb{Z}} 2^{\alpha l} \max \left\{2^{\beta l}, 2^{-n l}\left|\xi_{3}\right|\right\}^{-1}+\sum_{l \in \mathbb{Z}} 2^{\alpha l} \max \left\{2^{\beta l}, 2^{-n l}\left|\xi_{3}\right|\right\}^{-1 / 2} \\
& \lesssim \sum_{2^{l} \geq\left|\xi_{3}\right|^{1 /(\beta+n)}} 2^{\alpha l} 2^{-\beta l}+\sum_{2^{l} \leq\left|\xi_{3}\right|^{1 /(\beta+n)}} 2^{\alpha l} 2^{n l}\left|\xi_{3}\right|^{-1} \\
&+\sum_{2^{l} \geq\left|\xi_{3}\right|^{1 /(\beta+n)}} 2^{\alpha l^{-1} 2^{-\frac{\beta}{2} l}+} \sum_{2^{l} \leq\left|\xi_{3}\right|^{1 /(\beta+n)}} 2^{\alpha l} 2^{\frac{n}{2} l}\left|\xi_{3}\right|^{-1 / 2} \\
& \lesssim\left|\xi_{3}\right|^{-\frac{\beta / 2-\alpha}{n+\beta}} \approx|\xi|^{-\frac{\beta / 2-\alpha}{n+\beta}} .
\end{aligned}
$$


(ii) $\left|\xi_{3}\right|<\left|\xi^{\prime}\right|$ :

$$
\begin{aligned}
\sum_{l \in \mathbb{Z}}\left|m_{l}(\xi)\right| \leq & \sum_{l \in \mathbb{Z}} \sum_{\boldsymbol{j} \in \mathcal{I}_{r} \cup \mathcal{I}_{\theta}}\left|m_{l, j}(\xi)\right|+\sum_{l \in \mathbb{Z}} \sum_{\boldsymbol{j} \in \mathcal{I}_{r r} \cup \mathcal{I}_{\theta \theta}}\left|m_{l, j}(\xi)\right| \\
& \lesssim \sum_{j \in \mathcal{I}_{r} \cup \mathcal{I}_{\theta}} \sum_{l \in \mathbb{Z}} 2^{\alpha l} \lambda^{-1}+\sum_{\boldsymbol{j} \in \mathcal{I}_{r r} \cup \mathcal{I}_{\theta \theta}} \sum_{l \in \mathbb{Z}} 2^{\alpha l} \lambda^{-1 / 2} \\
& \lesssim \sum_{l \in \mathbb{Z}} 2^{\alpha l} \max \left\{2^{\beta l}, 2^{-l}\left|\xi^{\prime}\right|\right\}^{-1}+\sum_{l \in \mathbb{Z}} 2^{\alpha l} \max \left\{2^{\beta l}, 2^{-l}\left|\xi^{\prime}\right|\right\}^{-1 / 2} \\
& \lesssim \sum_{2^{l} \geq\left|\xi^{\prime}\right|^{1 /(\beta+1)}} 2^{\alpha l} 2^{-\beta l}+\sum_{2^{l} \leq\left|\xi^{\prime}\right|^{1 /(\beta+1)}} 2^{\alpha l} 2^{l}\left|\xi^{\prime}\right|^{-1} \\
& +\sum_{2^{l} \geq\left|\xi^{\prime}\right|^{1 /(\beta+1)}} 2^{\alpha l} 2^{-\frac{\beta}{2} l}+\sum_{2^{l} \leq\left|\xi^{\prime}\right|^{1 /(\beta+1)}} 2^{\alpha l} 2^{\frac{1}{2} l}\left|\xi^{\prime}\right|^{-1 / 2} \\
\lesssim & \left|\xi^{\prime}\right|^{-\frac{\beta / 2-\alpha}{1+\beta}} \approx|\xi|^{-\frac{\beta / 2-\alpha}{1+\beta}} .
\end{aligned}
$$

Therefore,

Corollary 3.2. If $\beta>2 \alpha>0$, then $\mathcal{R}$ is bounded from $L^{2}$ to $L_{s}^{2}$ for all $s \leq \frac{\beta / 2-\alpha}{n+\beta}$.

For $L^{p}$ Sobolev estimate, define

$$
\mathcal{R}_{z} f(x):=\left(\langle\xi\rangle^{z} m \widehat{f}\right)^{\vee}(x), \quad\langle\xi\rangle=\left(1+|\xi|^{2}\right)^{1 / 2} .
$$

Note that for $\operatorname{Re}(z)=0 \mathcal{R}_{z}=\mathcal{R}$, which has $L^{p}$ range of 3.19 . Then, $\left\{\mathcal{R}_{z}\right\}$ is an analytic family of linear operators of admissible growth defined in the strip $S:=\{z \in \mathbb{C} \mid 0 \leq$ $\left.\operatorname{Re}(z) \leq s_{1}=\frac{\beta / 2-\alpha}{n+\beta}\right\}$. Therefore, using interpolation of analytic families of operators (L. Grafakos [2, 1.3.3]), one has

Corollary 3.3. If $\beta>2 \alpha>0$ and $\frac{\alpha}{\beta}+\frac{s(n+\beta)}{\beta}<\frac{1}{p}<\frac{\beta-\alpha}{\beta}-\frac{s(n+\beta)}{\beta}$, then $\mathcal{R}$ is bounded from $L^{p}$ to $L_{s}^{p}$ for all $s \leq s_{1}=\frac{\beta / 2-\alpha}{n+\beta}$.

\section{Necessity of $L^{2}$ estimates}

Consider the case of $l>0$, since for $l \leq 0$ our multiplier $m$ only depends on $\alpha>0$. In addition, assume that $\frac{\Omega(t)}{|t|^{n}}=\frac{t_{n}}{|t|^{n+1}}$ with vector $\xi^{\prime}=\left(0, \ldots, \xi_{n}\right)$ fixed. Without loss of generality, one takes the corresponding part of the multiplier $m$ as

$$
m_{+}(\xi):=\int_{S^{n-2}} \int_{0}^{\pi} \int_{0}^{1} e^{-2 \pi i\left[r\left|\xi^{\prime}\right| \cos (\theta)+\xi_{n+1} r^{k}+r^{-\beta}\right]} \cos (\theta)(\sin (\theta))^{n-2} r^{-\alpha-1} d r d \theta d \sigma .
$$

Now, switch the order of the integrals so that one can extract the term independent of $\theta$ from the original integrand as follows:

$$
\int_{0}^{1} e^{-2 \pi i\left[\xi_{n+1} r^{k}+r^{-\beta}\right]} r^{-\alpha-1} \int_{S^{n-2}} \int_{0}^{\pi} e^{-2 \pi i r\left|\xi^{\prime}\right| \cos (\theta)} \cos (\theta)(\sin (\theta))^{n-2} d \theta d \sigma d r,
$$


and set

$$
G\left(r\left|\xi^{\prime}\right|\right):=\left|S^{n-2}\right| \int_{0}^{\pi} e^{-2 \pi i r\left|\xi^{\prime}\right| \cos (\theta)} \cos (\theta)(\sin (\theta))^{n-2} d \theta
$$

So,

$$
m_{+}(\xi)=\int_{0}^{1} e^{-2 \pi i\left[\xi_{n+1} r^{k}+r^{-\beta}\right]} r^{-\alpha-1} G\left(r\left|\xi^{\prime}\right|\right) d r .
$$

Then, use integration by parts, change of variables $2 \pi r\left|\xi^{\prime}\right|=x,-\cos (\theta)=t$, and (2.1) so that $G\left(r\left|\xi^{\prime}\right|\right)$ can be written as

$$
\begin{aligned}
G\left(r\left|\xi^{\prime}\right|\right) & =\left|S^{n-2}\right|\left[\left.\frac{e^{-2 \pi i r\left|\xi^{\prime}\right| \cos (\theta)}(\sin \theta)^{n-1}}{n-1}\right|_{\theta=0} ^{\theta=\pi}-\frac{2 \pi i r\left|\xi^{\prime}\right|}{n-1} \int_{0}^{\pi} e^{-2 \pi i r\left|\xi^{\prime}\right| \cos \theta}(\sin \theta)^{n} d \theta\right] \\
& =\left|S^{n-2}\right| \frac{i x}{1-n} \int_{-1}^{1} e^{i x t}\left(1-t^{2}\right)^{(n-1) / 2} d t
\end{aligned}
$$

Now, with $\left|S^{n-2}\right|=\frac{2 \pi^{n / 2}}{\Gamma(n / 2-1 / 2) \pi^{1 / 2}}$ and the last equality of $(2.1)$, one has

$$
G\left(r\left|\xi^{\prime}\right|\right)=\frac{-2 \pi i}{\left(r\left|\xi^{\prime}\right|\right)^{n / 2-1}} J_{n / 2}\left(2 \pi r\left|\xi^{\prime}\right|\right) .
$$

So,

$$
\left|m_{+}(\xi)\right|=2 \pi\left|\int_{0}^{1} e^{-2 \pi i \phi_{1}(r)} \frac{J_{n / 2}\left(2 \pi r\left|\xi^{\prime}\right|\right)}{\left(r\left|\xi^{\prime}\right|\right)^{n / 2-1}} \frac{1}{r^{\alpha+1}} d r\right|,
$$

where $\phi_{1}(r)=\xi_{n+1} r^{k}+r^{-\beta}$.

Actually,

$$
\begin{aligned}
& \phi_{1}^{\prime}(r)=\xi_{n+1} k r^{k-1}-\beta r^{-\beta-1}, \\
& \phi_{1}^{\prime \prime}(r)=\xi_{n+1} k(k-1) r^{k-2}+\beta(\beta+1) r^{-\beta-2}>0, \quad \forall r \in(0, \tau) .
\end{aligned}
$$

With 4.2 and $\phi_{1}(r)$ tends to $\infty$ as $r \rightarrow 0$, note that the $r_{0}=\left(\frac{\beta}{\xi_{n+1} k}\right)^{\frac{1}{\beta+k}}$ is the only critical point of $\phi_{1}(r)$.

Then, write (4.1) as

$$
\int_{0}^{1}=\int_{0}^{a}+\int_{a}^{b}+\int_{b}^{1}=I I I+I+I I,
$$

where $a=\frac{1}{2} r_{0}$, and $b=\frac{3}{2} r_{0}$; it is no harm since $\tau \rightarrow \infty$.

Now, choose $\xi$ so that $\left|\xi^{\prime}\right|=\frac{\tau}{2 \pi}$ and $\xi_{n+1}=\frac{\beta}{k} \tau^{\beta+k}$ for $\tau>0$, so that

$$
\begin{aligned}
r_{0} & =\tau^{-1}, \\
\frac{J_{n / 2}\left(2 \pi r\left|\xi^{\prime}\right|\right)}{\left(r\left|\xi^{\prime}\right|\right)^{n / 2-1}} & =C(n) \frac{J_{n / 2}(\tau r)}{(\tau r)^{n / 2-1}}, \\
\phi_{1}(r) & =\frac{\beta}{k} \tau^{\beta+k} r^{k}+r^{-\beta} .
\end{aligned}
$$


Case I: Since $r_{0}$ tends to 0 as $\tau \rightarrow \infty$, use change of variable $r \rightarrow r_{0} s$ which is equivalent to $s=\tau^{-1} r$ so that

$$
\int_{a}^{b}=\tau^{\alpha} e^{2 \pi i \tau^{-\beta} \phi_{1}\left(r_{0}\right)} \int_{1 / 2}^{3 / 2} e^{-2 \pi i \tau^{\beta}\left[\tau^{-\beta}\left(\phi_{1}\left(r_{0} s\right)-\phi_{1}\left(r_{0}\right)\right)\right]} \frac{J_{n / 2}(s)}{s^{n / 2}} \frac{1}{s^{\alpha}} d s,
$$

and we set $\Phi(s)=\tau^{-\beta}\left(\phi_{1}\left(r_{0} s\right)-\phi_{1}\left(r_{0}\right)\right)$ and note that

$$
\begin{gathered}
\Phi(1)=0, \quad \Phi^{\prime}(1)=0, \\
\Phi^{\prime \prime}(1)=\tau^{-\beta} \phi_{1}^{\prime \prime}\left(\tau^{-1}\right) \tau^{-2}=\tau^{-\beta-2}\left[\beta(k-1) \tau^{\beta+2}+\beta(\beta+1) \tau^{\beta+2}\right]=\beta(\beta+k)>0
\end{gathered}
$$

by 4.2, 4.3), 4.4). Then, since $\frac{J_{n / 2}(s)}{s^{n / 2}} \frac{1}{s^{\alpha}}$ is smooth in $[1 / 2,3 / 2]$, one can show, using Lemma 2.2 with $\lambda=\tau^{\beta}$, that the estimate of $I$ is given by

$$
|I| \sim \tau^{\alpha-\beta / 2} \quad \text { as } \tau \rightarrow \infty
$$

which is bounded if $\beta \geq 2 \alpha$.

Case II: In case $I I$, we set $r \rightarrow \tau^{-1} r$ so that

$$
I I=\tau^{\alpha} \int_{3 / 2}^{\tau} e^{-2 \pi i \phi_{1}\left(\tau^{-1} r\right)} \frac{J_{n / 2}(r)}{r^{n / 2}} \frac{1}{r^{\alpha}} d r,
$$

and $\phi_{1}\left(\tau^{-1} r\right)=\tau^{\beta}\left(\frac{\beta}{k} r^{k}+r^{-\beta}\right)$. Let $\phi_{2}=\frac{\beta}{k} r^{k}+r^{-\beta}$ then $\phi_{2}^{\prime}(r)$ increases monotonically so that $\left|\phi_{2}^{\prime}(r)\right| \geq\left|\phi_{2}^{\prime}(b)\right|=\beta\left[\left(\frac{3}{2}\right)^{k-1}-\left(\frac{2}{3}\right)^{\beta+1}\right]>0, \forall r \in[3 / 2, \tau]$. So, if $\psi(r)=\frac{J_{n / 2}(r)}{r^{n / 2}} \frac{1}{r^{\alpha}}$, then

$$
|I I| \leq C(n, \beta, k) \tau^{\alpha-\beta}\left[|\psi(\tau)|+\int_{3 / 2}^{\tau}\left|\psi^{\prime}(t)\right| d t\right]
$$

by Lemma 2.1 with $\lambda=\tau^{\beta}$. Since $|\psi(\tau)| \rightarrow 0$ as $\tau \rightarrow \infty$, one has

$$
\leq C^{\prime}(n, \beta, k) \tau^{\alpha-\beta} \int_{3 / 2}^{\tau}\left|\psi^{\prime}(t)\right| d t
$$

Also, since $\left|\psi^{\prime}(r)\right|=\left|\frac{J_{n / 2+1}(r)}{r^{n / 2+\alpha}}+\frac{J_{n / 2}(r)}{r^{n / 2+\alpha+1}}\right|$, it follows that

$$
\left|\psi^{\prime}(r)\right| \lesssim\left|\frac{1}{r^{n / 2+1 / 2+\alpha}}\right|+\left|\frac{1}{r^{n / 2+1 / 2+\alpha+1}}\right|
$$

by Lemma 2.3. Then, with $n \geq 2$,

$$
|I I| \lesssim C(n, \beta, k, \alpha) \tau^{\alpha-\beta} \quad \text { as } \tau \rightarrow \infty
$$

which is bounded if $\beta \geq \alpha$.

Case III: As in case $I, I I$, by the change of variable, $r \rightarrow \tau^{-1} r$, so that

$$
I I I=\tau^{\alpha} \int_{0}^{1 / 2} e^{-2 \pi i \tau^{\beta} \phi_{2}(r)} \psi(r) d r
$$


where we have $\left|\phi_{2}^{\prime}(r)\right| \geq \beta\left(2^{\beta+1}-2^{1-k}\right)$ for $r \in[0,1 / 2]$. Then integration by parts gives

$$
\begin{aligned}
& |I I I| \leq \tau^{\alpha}\left[|F(r) \psi(r)|_{r=0}^{r=1 / 2}|+| \int_{0}^{1 / 2} F(r) \frac{d}{d r} \psi(r) d r \mid\right]=\tau^{\alpha}\left[\left|I I I_{1}\right|+\left|I I I_{2}\right|\right], \\
& F(r)=\int_{0}^{r} e^{-2 \pi i \tau^{\beta} \phi_{2}(t)} d t, \quad r \leq \frac{1}{2} .
\end{aligned}
$$

Since $|F(r)| \leq C(\beta, k) \tau^{-\beta}$ by Lemma 2.1 with $\lambda=\tau^{\beta}$, and $J_{m}(r) \sim r^{m}$ as $r \rightarrow 0$ for $\operatorname{Re}(m)>-1 / 2$,

$$
\begin{aligned}
\tau^{\alpha}\left|I I I_{1}\right| & \leq \tau^{\alpha}|F(1 / 2)||\psi(1 / 2)| \\
& \leq C_{\beta, k} \tau^{\alpha} \tau^{-\beta}\left|J_{n / 2}(1 / 2)\right| 2^{\alpha+n / 2} \sim \tau^{\alpha-\beta}
\end{aligned}
$$

which is bounded if $\beta>\alpha$. For $I I I_{2}$, note that $\left|\phi_{2}^{\prime}(r)\right| \geq \frac{1}{2} \beta r^{-\beta-1}$ if $r \in\left[0,\left(\frac{1}{2}\right)^{\frac{1}{\beta+k}}\right]$ and if $k \geq 1$, then $\left(\frac{1}{2}\right)^{\frac{1}{\beta+k}}>\frac{1}{2}$; that is, $|F(r)| \leq C(\beta, k) \tau^{-\beta} r^{\beta+1}$ in $[0,1 / 2]$. Then, by Lemma 2.3 .

$$
\begin{aligned}
\left|I I I_{2}\right| & \leq \int_{0}^{1 / 2}|F(r)|\left[r^{-n / 2}\left|J_{n / 2+1}(r)\right| \frac{1}{r^{\alpha}}+\frac{\left|J_{n / 2}(r)\right|}{r^{n / 2}} \frac{\alpha}{r^{\alpha+1}}\right] d r \\
& \leq C_{\beta, k}^{\prime} \tau^{-\beta} \int_{0}^{1 / 2} \frac{\left|J_{n / 2+1}(r)\right|}{r^{n / 2}} r^{1+\beta-\alpha}+\frac{\left|J_{n / 2}(r)\right|}{r^{n / 2}} \alpha r^{\beta-\alpha} d r \\
& \sim \tau^{-\beta}\left[\int_{0}^{1 / 2} r^{2+\beta-\alpha} d r+\int_{0}^{1 / 2} r^{\beta-\alpha} d r\right] \quad \text { as } \tau \rightarrow \infty .
\end{aligned}
$$

Both integrals are bounded if $2+\beta-\alpha>-1$ and $\beta-\alpha>-1$ respectively; hence the entire term is bounded if $\beta>\alpha-1$. Thus,

$$
\tau^{\alpha}\left|I I I_{2}\right| \leq C_{\alpha, \beta, k} \tau^{\alpha-\beta} \quad \text { as } \tau \rightarrow \infty
$$

which is bounded if $\beta \geq \alpha$.

By 4.5-4.7), it follows that $I I$ and $I I I$ decay faster than $I$ does as $\tau \rightarrow \infty$. Thus one can conclude that

$$
\left|m_{+}(\xi)\right| \sim \tau^{\alpha-\beta / 2} \quad \text { as } \tau \rightarrow \infty
$$

along $\xi=\left(\xi^{\prime}, \frac{\beta}{k}\left(2 \pi\left|\xi^{\prime}\right|\right)^{\beta+k}\right)$ with $\left|\xi^{\prime}\right|=\frac{\tau}{2 \pi}$; note that the direction of $\xi^{\prime}$ is fixed. Hence we have the following

Theorem 4.1. Suppose that $\xi^{\prime}=\tau u$ for $\tau>0$ and let a fixed $u \in S^{n-1}$. Then along $\xi=$ $\left(\xi^{\prime}, \frac{\beta}{k}\left(2 \pi\left|\xi^{\prime}\right|\right)^{\beta+k}\right)$, the Fourier multiplier, $m(\xi)$, of $\mathcal{R}$ associated to the kernel $\frac{\Omega(t)}{|t|^{n}}=\frac{t_{n}}{|t|^{n+1}}$ satisfies that $|m(\xi)| \sim_{\alpha, \beta, k} \tau^{-(\beta / 2-\alpha)}$ as $\tau \rightarrow \infty$.

This proves that the operator class $\{\mathcal{R}\}$ has a necessary condition, $\beta \geq 2 \alpha>0$, for $L^{2}$ boundedness. In other words, if $\beta<2 \alpha$, then there exists an operator $\mathcal{R}$ of the form (1.1) which is not bounded on $L^{2}$. 


\section{References}

[1] S. Chandarana, $L^{p}$-bounds for hypersingular integral operators along curves, Pacific J. Math. 175 (1996), no. 2, 389-416.

[2] L. Grafakos, Classical Fourier Analysis, Second edition, Graduate Texts in Mathematics 249, Springer, New York, 2008.

[3] N. Laghi and N. Lyall, Strongly singular integrals along curves, Pacific J. Math. 233 (2007), no. 2, 403-415.

[4] E. M. Stein, Harmonic Analysis: Real-variable methods, orthogonality, and oscillatory integrals, Princeton Mathematical Series 43, Monographs in Harmonic Analysis III, Princeton University Press, Princeton, NJ, 1993.

[5] E. M. Stein and S. Wainger, Problems in harmonic analysis related to curvature, Bull. Amer. Math. Soc. 84 (1978), no. 6, 1239-1295.

[6] X. Wu and X. Yu, Strongly singular integrals along curves on $\alpha$-modulation spaces, J. Inequal. Appl. 2017 (2017), 185-197.

Jin Bong Lee, Jongho Lee and Chan Woo Yang

Department of Mathematics, Korea University, Seoul 136-701, Republic of Korea

E-mail address: 9h0st@korea.ac.kr, 1jh0308@korea.ac.kr, cw_yang@korea.ac.kr 